\title{
EchoGéo
}

$58 \mid 2021$

Varia

\section{Attention : projet ! Comprendre les métiers de l'urbanisme par la fiction documentaire}

Entretien avec Elsa Vivant, réalisé par Serge Weber le 8 décembre 2021 à Paris

\section{Elsa Vivant et Serge Weber}

\section{OpenEdition}

Journals

Édition électronique

URL : https://journals.openedition.org/echogeo/22587

DOI : $10.4000 /$ echogeo.22587

ISSN : 1963-1197

Éditeur

Pôle de recherche pour l'organisation et la diffusion de l'information géographique (CNRS UMR 8586)

Référence électronique

Elsa Vivant et Serge Weber, «Attention : projet ! Comprendre les métiers de l'urbanisme par la fiction documentaire », EchoGéo [En ligne], 58 | 2021, mis en ligne le 31 décembre 2021, consulté le 14 février 2022. URL : http://journals.openedition.org/echogeo/22587; DOI : https://doi.org/10.4000/echogeo. 22587

Ce document a été généré automatiquement le 14 février 2022.

EchoGéo est mis à disposition selon les termes de la licence Creative Commons Attribution - Pas d'Utilisation Commerciale - Pas de Modification 4.0 International (CC BY-NC-ND) 


\section{Attention : projet ! Comprendre les métiers de l'urbanisme par la fiction documentaire}

Entretien avec Elsa Vivant, réalisé par Serge Weber le 8 décembre 2021 à Paris

\section{Elsa Vivant et Serge Weber}

\section{RÉFÉRENCE}

Elsa Vivant

L'impasse. Scènes de l'urbanisme ordinaire

2021. Créaphis Éditions, 213 p.

1 Sociologue et urbaniste, Elsa Vivant est surtout connue pour ses travaux sur les rapports entre les transformations urbaines et les milieux artistiques et culturels (2020). En parallèle de ses recherches sur des terrains variés, elle s'interroge depuis plusieurs années sur les évolutions des métiers de l'urbanisme et sur les trajectoires professionnelles des diplômés (2014). Le livre qu'elle propose distille des années d'enquêtes empiriques approfondies et d'interrogations réflexives sur les contradictions auxquelles sont confrontées toutes les catégories d'acteurs impliqués dans les projets urbains et plus généralement dans la production urbaine. C'est un ouvrage assez inclassable à première vue, puisqu'il n'est écrit ni comme un texte académique, ni comme un essai. On pourrait l'apparenter à une fiction documentaire. C'est ce pas de côté dans l'énonciation, ouvertement fictionnelle, qui lui permet de faire vivre la réalité des situations professionnelles, parfois éprouvantes, de divers métiers.

Serge Weber (SW). La lecture de votre ouvrage est saisissante, tant par sa forme, tout à fait nouvelle en sciences sociales, que par son contenu. Vous avez choisi de proposer une fiction, certes, mais une fiction qui amène le lecteur à faire un plongeon dans le cœur du réel, un réel qui vous prend à la gorge. II est donc avéré que l'écriture fictionnelle, à partir 
d'un matériau empirique de recherche de terrain au long cours réussit à s'approcher au plus près de la réalité.

Elsa Vivant (EV). En pensant aux personnes qui n'ont pas de familiarité avec ce milieu, j'ai souhaité parler de la diversité des métiers qui sont derrière la prise de décision dans les projets de transformation urbaine. On parle toujours des élus, des concepteurs et de choses qui restent finalement assez abstraites. Il me semblait nécessaire de restituer les réalités vécues par les personnes qui travaillent au quotidien et qui font face à des décisions qui, pour certaines, ne font pas sens ou sont inapplicables. C'était une des intentions initiales. Je voulais aussi montrer comment, parfois voire souvent, cette réalité conduit à des situations de mal-être au travail. À force d'enquêter auprès d'acteurs de la production urbaine, j'avais envie de raconter le quotidien de ces profesionnel-les, qui sont les personnages principaux du livre, en incluant une enseignante-chercheuse en urbanisme. Celle-ci est elle-même confrontée à des décisions qui lui échappent, qui ne font pas sens et rendent son travail plus complexe. Il fallait sortir de la division sectorielle et rendre compte d'une complexification, complexification qui ne concerne pas seulement l'aménagement, mais qu'on retrouve dans d'autres domaines professionnels.

Il faut s'imaginer tout ce qui s'entrecroise et s'articule quand on parle d'urbanisme où chaque décision peut avoir des effets en cascade. La complexification de ce qu'on appelle le jeu d'acteurs et l'externalisation de nombreuses tâches a pour corollaire la spécialisation des expertises et, en miroir, la managerialisation du projet, au détriment d'une vision systémique et parfois du sens même de l'action urbaine ${ }^{1}$. Et cela aussi, c'est un facteur de fragilité.

SW. Comment avez-vous construit votre texte? Je vous propose de commencer par votre choix de recourir tout au long à plusieurs registres d'énonciation bien différenciés.

EV. À l'origine, j'avais envie d'écrire un roman, à la manière de la Vingt-septième ville de Jonathan Franzen (2005). Cela s'est avéré très difficile par rapport à une écriture démonstrative académique, qui peut difficilement rendre un roman intéressant. La deuxième difficulté tenait au trop-plein de matière à agencer et à mon envie d'aborder un grand nombre de thèmes. L'idée m'est venue en faisant un plan, un dessin, que je n'ai pas inclus dans le livre, une sorte de carte avec des situations territoriales, des références auxquelles je pensais, des personnages qui pouvaient incarner le thème ou l'enjeu. Le point de départ, c'est donc une carte, un peu abstraite, pour commencer à établir de quoi j'avais envie de parler et créer des mises en relation : une carte géographique et mentale. L'étape suivante a été d'organiser le matériau par thèmes, pour stucturer le texte au fur et à mesure. Chaque grande thématique se déroule au gré de quatre saisons, de l'automne à l'été, qui permettent de structurer l'avancée de l'intrigue, car il s'agit bien d'une intrigue, vers sa résolution. Ensuite, je me suis rendu compte que raconter l'histoire en tant que telle, ce n'était pas le plus intéressant. Ce qui faisait sens, c'était plutôt tous les fragments de réel, l'envie de raconter des scènes, des situations, d'intégrer des documents collectés au cours de la recherche, à glisser tels quels dans le texte. Progressivement, c'est devenu un agencement de matériaux divers plus qu'un récit linéaire. Ce qui correspond aussi au propos de fond: dans le monde professionnel, «les choses se fragmentent ». Le texte est fragmenté, avec des régimes d'énonciation différents, pour traduire la fragmentation de l'organisation de la production urbaine. 
2 SW. En effet, il y a des extraits de carnets de terrain, des passages narratifs, des conversations entendues, des verbatims de réunions, des SMS, des documents, et même quelque chose qui ressemble à une pièce de théâtre, sachant que vous avez déjà abordé ce thème (Vivant, 2021). Prenons par exemple les carnets pour commencer, il m'a semblé qu'ils servaient de fil conducteur et de repère tout du long.

EV. Ces carnets sont ceux d'un personnage que je n'ai construit qu'à la fin, une enseignante-chercheuse qui fait une enquête sur un projet d'aménagement incluant un grand équipement. Mais la narratrice n'est pas l'auteure : ses sujets de recherche sont proches des miens, mais les autres dimensions de sa vie en font un personnage fictif. Pour autant, ce sont essentiellemement mes propres carnets d'enquête que j'ai adaptés pour qu'ils constituent un fil rouge. Ils exposent l'enquête et ses aléas : les lapins posés, les retards, l'enregistreur qui n'a plus de piles, les questionnements qui surgissent au fur et à mesure entre ce qu'on croit enquêter au début, le projet initial déposé pour obtenir un financement de recherche, et ce qui a été bousculé par l'enquête elle-même et la rencontre avec ces professionnels, leurs bureaux, ou encore les territoires sur lesquels ils interviennent, qui sont pour la plupart des quartiers populaires qui font l'objet d'un programme de rénovation urbaine. Dans le carnet de terrain, on a la liberté de noter ses états d'âme, ses remises en question, de reformuler la manière de se poser les problèmes. Les carnets exposent en fait les évolutions d'un questionnement d'une chercheuse face à ses propres difficultés scientifiques, professionnelles et personnelles. Il lui arrive des choses, qui arrivent aussi aux autres personnages. Tous sont confrontés à un même problème, celui du logement, même si ils le sont de manière différente. Tous ces personnages, tous ces professionnels, sont aussi des habitants. L'enseignante-chercheuse vit le déclasssement résidentiel: ses revenus d'universtaire ne lui permettent plus, aujourd'hui, d'accéder facilement à un logement dans une grande métropole. D'autres personnages vivent l'expérience de l'accession à la propriété et ses nouvelles responsabilités.

SW. Oui, ce qui m'a frappé, c'est la dimension technique et très matérielle des questions de logement pour tous les personnages. Mais il n'y a pas que cela: vous consignez d'autres expériences qui n'ont a priori rien à voir, comme cette exposition visitée, qui vous a inspiré des réflexions sur le «membre-fantôme ».

EV. C'est comme dans tout travail de recherche et de conceptualisation : il n'y a pas que les lectures académiques qui nous influencent, mais aussi des situations vécues, et bien sûr, le travail de certains artistes qui questionnent le contemporain. Le travail de Kader Attia ${ }^{2}$ sur le membre fantôme m'a beaucoup marquée. Je pense aussi à un livre sur une expérience menée par un enseignant d'histoire et géographie avec ses élèves, portant sur les mémoires résidentielles des familles. Il est apparu que dans certaines familles, il n'y avait aucune trace des logements occupés, qui tous ont été détruits par l'Etat, traduisant un rapport au logement dénigré et illégitime (Lepoutre, 2005). Dans les deux cas, la mémoire de la guerre d'Algérie peut faire réfléchir à de tout autres situations, comme par exemple, la politique de rénovation urbaine mise en œuvre depuis 2003. J'évoque Kader Attia car c'est vraiment très marquant, mais il y a eu d'autres œuvres d'artistes dont l'écriture documentaire contemporaine a été très stimulante, même si elles ne parlent pas de questions urbaines. Y faire référence est une manière d'expliquer pourquoi j'ai recours à cette forme littéraire non académique: par d'autres formes de représentations des enquêtes en sciences 
sociales, on peut exprimer des idées différemment mais aussi les faire surgir. C'est par le travail de la forme que certaines compréhensions des réalités observées peuvent apparaître.

SW. Sur ce thème du déni de reconnaissance et de mémoire, l'insert de l'extrait du texte de Colette Pétonnet (1968) résonne avec force. Évoquons maintenant les épisodes de narration: certains sont à la troisième personne, comme dans un roman, d'autres à la première comme une autofiction. J'ai eu l'impression que le personnage de Fred, la consultante qui répond à l'appel d'offre, était le symétrique de Julie, l'enseignantechercheuse.

EV. Les narrations se font autour d'un ou plusieurs personnages. Elles sont écrites à partir des entretiens que j'ai faits avec des professionnels pendant cinq ou sept ans. Ce sont des réécritures d'extraits d'entretiens permettant de construire des idéauxtypes de professionnels qui sont incarnés par des anecdotes et détails personnels, dont le chargé de projet (Sylvain) et la consultante (Fred). J'ai beaucoup enquêté auprès de consultants ; certains travaillent en bureaux d'études et d'autres travaillent seuls, confrontés à la difficulté de gérer leur entreprise, ce qui ne s'apprend que sur le tas. Contrairement au chef de projet, ils ont la (relative) liberté de choisir leur sujet de travail : c'est à la fois plus instable et plus intéressant et motivant. Vient ensuite la chargée de relogement (Virginie), qui émane de trois entretiens avec des personnes qui ont suivi des ménages qui allaient être expulsés et relogés, et qui ont témoigné de l'impossibilité de répondre à toutes les demandes: elles sont confrontées à des situations de mal logement et de logement indigne auxquelles elles ne peuvent pas répondre, tout en contribuant à des pratiques parfois traumatiques d'expulsion et de déplacement, pour la réalisation des projets urbains. Elles ont des positions intéressantes d'un point de vue dramaturgique : elles sont porteuses de convictions (la défense d'un droit au logement de qualité pour tous) mais doivent faire avec une réalité tragique sur laquelle elles n'ont pas de prise (des gens à qui on ne trouve pas de solution) voire à laquelle elles participent.

Les maires sont évidemment présents. Il m'a semblé important d'avoir deux figures de maires, au risque d'être caricaturale. Certains élus sont des personnes investies, animées d'une vocation citoyenne, qui y mettent toute leur âme. Leur engagement dévore leur vie, mais leurs convictions sont fortes. D'autres s'engagent en politique pour atteindre une certaine position sociale, avec des convictions plus malléables, moins construites. Ces façons d'être "élu » ont des effets sur les administrations: quand on est porté par des équipes municipales et des maires qui s'intéressent aux projets, qui se forment, qui ont une vision et suscitent l'adhésion, cela rend plus acceptable une charge de travail intense. Inversement, quand on est en désaccord avec des élus qui ne sont pas au bon endroit vis-à-vis des services, cela peut déraper, cela peut se traduire par des déstabilisations des équipes. Un changement politique, c'est toujours un moment délicat. J'avais très envie de le raconter, car on n'en parle jamais! On parle des élus mais pas des personnes qui travaillent dans les services muncipaux. Et ce n'est pas qu'une question de positionnement idéologique, loin de là, il s'agit plutôt des manières de se positionner par rapport aux administrations et aux habitants. Ces figures ont été inspirées d'entretiens que j'ai pu faire ou de récits recueillis. 


\section{SW. Les conversations entendues?}

EV. Elles ont été réellement entendues! Avec le téléphone portable, l'intimité des uns et des autres s'expose dans l'espace public réel, pas seulement virtuel, avec un effet assez étrange. J'ai noté des choses qui résonnaient étonnamment avec l'enquête. Je pensais au film Bird People de Pascale Ferran ${ }^{3}$. La première scène se déroule dans une rame de RER, on entend les pensées des voyageurs et ce que les gens font sur leur téléphone.

SW. Ces conversations m'ont semblé introduire ce que l'enquête laisse hors champ, à savoir les mobilités quotidiennes, les rythmes de vie vécue, révélateurs de fortes différenciations sociales.

EV. Oui, par exemple cette femme qui est contrainte de refuser un emploi intérimaire de trois heures loin de chez elle, ce qui est inconciliable avec ses temps de transports et ses contraintes familiales. Si on tend l'oreille, on n'entend pas la même chose d'un transport à l'autre : il y a une géographie sociale des conversations dans le métro. Le territoire du roman, il faut le penser comme une chimère: c'est un montage de plusieurs situations observées à différents endroits. Les conversations téléphoniques sont aussi un moyen de nous faire voyager entre plusieurs espaces. À certains moments, j'ai eu le sentiment désagréable d'écouter aux portes; mais non, avec les usages du téléphone, l'intimité est imposée et exposée à tous.

\section{SW. Les documents insérés ? Certains sont particulièrement frappants.}

EV. Il s'agit de vrais documents, ils n'ont pas été inventés, simplement un peu modifiés pour les anonymiser, en faisant notamment disparaître les noms de lieux. Il fallait leur donner une forme qui exprime clairement qu'ils sont, à la différence du reste du texte, réels: les pages sont grises. C'est aussi la forme choisie pour les extraits d'ouvrages connus et identifiés. Les pages sur fond blanc sont, à l'inverse, d'autres registres d'écriture : cela imposait un choix de mise en forme différenciée. J'ai sélectionné des documents qui ont une force à la fois politique et poétique : des lettres d'injonction à un promoteur, la lettre du maire d'une commune où on attend toujours le métro alors qu'il était déjà projeté dans les années 1960, les lettres de plaintes de riverains. Dans les services, les lettres de plaintes, anonymes ou non, sont le quotidien. Certaines sont mêmes assez drôles. Je me suis aussi essayée à des exercices stylistiques dont la dimension poétique fait ressortir l'enjeu politique. Par exemple en synthétisant, par une succession de phrases nominales courtes, un entretien très intéressant avec un architecte urbaniste d'une grande opération de rénovation urbaine. J'observe sa réflexivité s'exercer au cours de l'entretien. Au début, il est très enthousiaste d'avoir participé à une grande aventure de rénovation urbaine. Progressivement, il semble prendre conscience de la violence symbolique que représentent les expulsions, si on considère qu'un "projet ambitieux» est un projet « qui démolit beaucoup ». J'ai transformé cet entretien en forme poétique avec une réflexion sur la langue. D'autres exercices de style essaient de mettre en évidence l'absurdité de certaines situations réelles, par exemple avec un calligramme. Quand un chef de projet passe son temps à coordonner des études sur beaucoup de sujets différents, il passe plus de temps à gérer les marchés publics qu'à faire le travail de fond. À force d'externaliser ce travail de fond à des personnes elles-mêmes happées par les contraintes de gestion, celui-ci passe au second plan, au détriment la mise en cohérence de toutes les études que le chef de projet a commanditées. 
SW. Les réunions, avec en parallèle des SMS échangés par les participants, sont particulièrement savoureuses! Et un peu tristes, car vous évoquez de façon subtile l'absence dans la présence, le défaut d'attention à la parole de l'autre.

EV. Dès le début je voulais restituer ce très vif sentiment que j'ai en assistant à des réunions : les participants n'écoutent pas, ils sont absents, ils sont ailleurs via leur téléphone ou leur ordinateur. Or, plus on se disperse plus on s'épuise. C'est sans doute pourquoi les réunions s'étirent en logueur. J'ai restitué une réunion à laquelle j'ai assisté, où personne ne disait rien à par le chef de projet et, à chaque fois que je repérais quelqu'un qui utilisait son téléphone (c'est-à-dire tout le temps), je le consignais sur la carte des participants que j'avais dressée. À partir de cela, j'ai réécrit un extrait de réunion, à l'ouverture de chaque saison, en inventant ce qui pouvait bien se passer sur l'écran des téléphones portables. J'ai dû faire un effort d'imagination car je n'ai pas de smartphone!

Les participants ont une vie parallèle à la réunion: cela crée une situation proprement théatrale, que j'imagine dans une forme de mise en scène.

SW. Vous l'écrivez explicitement, le scénario de la mise en scène.

EV. Cette idée m'est venue en voyant certaines créations de l'Encyclopédie la parole une compagnie qui travaille beaucoup sur les sons, qui collecte des sons médiatiques, les monte, les met en scène avec des dispositifs différents. Dans Suite $n^{\circ} 2$, on voyait à la fois des gens qui parlaient devant un pupitre et des textes projetés sur le fond. Le spectateur, face au brouhaha, doit se laisser porter par la musicalité. On m'a parlé d'un autre spectacle, que je n'ai pas vu, où étaient projetés les profils facebook des spectateurs présents dans la salle (situation que j'évoque dans le texte), créant une mise en abyme de l'intime exposé sur les réseaux sociaux, c'est ce qu'on peut appeler l'extime. Le dispositif scénique rend visible cette dilution des sphères publique et privée.

SW. Est-ce que les quelques pages de dialogues avec didascalies sont une manière de pièce de théatre?

EV. Je n'y avais pas pensé. Pour moi, ces personnes sont toutes au téléphone, pendant un moment très particulier. C'est la visite d'un président de la République à laquelle j'ai assisté. C'était assez amusant de voir la mise en scène du pouvoir au cours de sa visite dans un territoire populaire : les rues sont bloquées, il y a de longs moments d'attente, le site est inaccessible pour les riverains, tout le monde est au téléphone pour trouver un moyen de s'approcher du président. Le lendemain, dans le train, je me mets écrire la scène sous la forme de conversations téléphoniques, certaines entendues, d'autres étaient des observations que je transforme en conversation, par exemple pour la personne qui ne parvient pas à rentrer chez elle, le chef de projet qui s'occupe de la rénovation urbaine et se rend compte qu'elle a finalement abouti à mettre des barrières partout.

SW. La tour de bureaux aujourd'hui démolie est un personnage elle aussi, finalement. Vous lui consacrez même une page à la première personne.

EV. Je vois progressivement, tout au long de l'enquête, sa démolition se faire, je la note à chaque visite dans mon carnet. Dans le cadre d'un autre travail, avec deux camarades et artistes, Karine Sahler et Clément Postec, nous avions mobilisé toutes les archives trouvées sur ce bâtiment et écrit sa biographie en inversant l'énonciation pour raconter l'histoire de son point de vue : le point de vue d'un bâtiment qui, à 40 ans, c'est à dire plutôt jeune, allait mourir. Ce texte, nous l'avions mis en scène dans 
la tour même, avant démolition, avec des habitants et des gens qui travaillent sur le site, tous ayant raconté ce qui s'était passé pour eux (des mariages, des souvenirs de la boîte de nuit fermée au début des années 2000, l'ambiance au travail). C'est un texte écrit dans un autre cadre, mais que j'ai remis ici car j'aime beaucoup cet exercice de décentrement.

SW. On voit en filigrane se dévoiler des formes de discrimination frappantes, comme si c'était un arrière-plan omniprésent, je pense notamment à un des personnages, ouvrier de chantier qui a subi une déqualification particulièrement forte.

EV. C'est sous-jacent, c'est un impensé, on pourrait faire une analyse intersectionnelle de l'urbanisme en tant que monde professionnel mais aussi en tant que mode de domination. Je ne vais pas le faire. J'ai été frappée par les rapports de genre entre les professionnels, mais aussi par la récurence de propos essentialistes énoncés presque sans vraiment y penser, sans se rendre compte de ce qu'ils véhiculent. À force d'être répétés, certains deviennent des légendes urbaines structurantes dans la légitimiation de l'action. Mais peut-être étais-je dans un état d'esprit d'hyper-vigilance à certains hiatus. Si je l'écrivais aujourd'hui, je parlerais davantage des questions de violence et de discrimination de genre, parce depuis ces enquêtes, des comportements ont été dénoncés publiquement et on ne peut plus être dans le déni. C'est quand même présent dans le texte, mais de manière subtile. Par exemple, l'enseignante-chercheuse rencontre un collègue plus âgé qui se comporte de façon très paternaliste. De même, les attentats de 2015 ont eu lieu pendant mon enquête et occupent une place particulière dans ce travail. Les personnes que j'ai rencontrées et qui travaillaient dans des quartiers populaires se posaient beaucoup de questions sur ce sujet au cours des entretiens. Elles s'interrrogeaient sur ce qu'elles font, ce qui n'a pas été fait, ce qui a été fait. Dans les territoires où j'ai enquêté, les problèmes étaient toujours définis soit par manque d'accès au transport soit par manque d'entretien du parc de logement. Ces focalisations occultent beaucoup d'autres dimensions, qui sont autant de leviers sur lesquels intervenir pour améliorer le quotidien des habitants. Pour mettre fin aux situations de misère auxquelles elle est confrontée, une des personnages en vient à comprendre que le nœud réside dans l'instabilité administrative que vivent les étrangers auprès desquels elle travaille. Ils sont confrontés au pouvoir discrétionaire des préfets et aux problèmes insolubles d'octroi de titres de séjour et de leur renouvellement; leur préoccupation première est de stabiliser leur situation administrative qui les entrave pour l'accès à un logement ou à un travail. Accorder des titres de séjours stables et sans conditions serait un moyen efficace pour sortir de nombreuses familles de la misère et pour lever les incertitudes et les vulnérabilités que l'instabilité administrative produit. D'autant plus que c'est très facile à mettre en œuvre, ce n'est pas cher, cela demande simplement un peu de volonté politique. Mais ça....

J'ai fait une visite d'un bâtiment qui va être exproprié dans le cadre d'un programme de rénovation urbaine, et j'ai été frappée par la diversité des logements. Entre un propriétaire qui avait passé toute ses économies et usé sa santé pour rénover et entretenir un logement devenu magnifique et un marchand de sommeil qui logeait huit personnes dans un dortoir de deux pièces, c'était le grand écart. Pour penser l'intervention dans des quartiers populaires qui ont été l'objet de nombreuses démolitions, il faut tenir avoir en tête ces complexités et certaines contradictions. Par exemple, un des immeubles qui ne devait pas être démoli a été évacué en urgence car 
sa façade s'est effondrée; alors qu'il est situé dans le périmètre d'une opération de rénovation urbaine de plus de 600 millions d'euros.

SW. En effet, sans dévoiler ici la résolution de l'intrigue, le choix du titre laisse penser que les contradictions grèvent beaucoup de choses, notamment votre belle phrase «que faire pour ceux qui tombent ? » face à « l'impossibilité de bien faire son travail »...

EV. Il y a quand même des résolutions de l'intrigue, à la fin, où chacun va trouver des manières de sortir des contradictions avec lesquelles il se débat. Mais c'est un phénomène transversal, qui ne se limite pas aux métiers de la ville. Qu'on travaille à France Télécom ou dans l'Education nationale, c'est pareil. À l'origine, je voulais faire quelque chose sur la souffrance au travail et un collègue m'a fait remarquer, avec raison, qu'on savait déjà tout: la psychodynamique du travail explique très bien l'enchainement des mécanismes qui causent de la souffrance au travail, qu'on pense aux études de Christophe Dejours (1993 [1980]) ou, plus récemment, au Travail disloqué de Guillaume Tiffon (2021). On le sait depuis longtemps, les procès ont eu lieu et, pour autant, on continue, on approfondit encore ces formes d'organisation du travail par projet qui génèrent des situations malsaines. Peut-être qu'en l'exprimant différement, on peut faire bouger les choses, dans tous les milieux professionnels. Le sens de ce livre, c'est de poser ce débat-là.

\section{BIBLIOGRAPHIE}

Bataille N., 2020. Experts et consultants au service de l'action publique locale: une approche pragmatique du travail de l'ingénierie privée. Thèse de doctorat en aménagement et urbanisme, Université de Tours.

Dejours C., 1993 [1980]. Travail : usure mentale. Paris, Bayard.

Franzen J., 2005. La vingt-septième ville [traduction française]. Paris, Seuil.

Gomes P., Pérès Y., 2021. Introducing real estate led start-up urbanism: An account from Greater Paris. Progress in Planning [En ligne], Elsevier, p. 100625. DOI: https://doi.org/10.1016/j.progress. 2021.100625

Lacroix G., 2019. Les équilibristes du développement durable. Une ethnographie des experts et de l'expertise en durabilité dans la fabrique urbaine. Thèse de doctorat en Architecture, aménagement de l'espace. Université Paris 8 Vincennes-Saint-Denis.

Lepoutre D., Cannoodt I., 2005. Souvenirs de familles immigrées. Paris, Odile Jacob.

Pétonnet C., 1968. Ces gens-là. Paris, Maspero.

Tiffon G., 2021. Le travail disloqué. Organisations liquides et pénibilité mentale du travail. Lormont, Le Bord de l'eau, coll. « Documents».

Vivant E., 2014. Jeunes urbanistes et auto-entrepreneur : un oxymore ? Les apprentissages d'une socialisation professionnelle atypique. In Mashino L., Scouarnec A. (dir.), Les Métiers de la Ville de Demain. Management Prospective Editions, p. 133-150. 
Vivant E., 2020. From margins to capital: The integration of spaces of artistic critique within capitalist urbanism. Journal of Urban Affairs, Wiley, p. 1-14. HAL Id: https://hal-enpc.archivesouvertes.fr/hal-02964262/document

Vivant E., 2021. Mettre en scène les territoires et leurs jeux d'acteurs par la création théâtrale. Annales de géographie, vol. 739-740, nº 3, p. 101-121.

\section{NOTES}

1. Plusieurs thèses ont mis cela en évidence, dont celles de Guillaume Lacroix (2019), Nicolas Bataille (2020) et celle, en cours, de Yoann Pérès (Gomes, Pérès, 2021).

2. Centre Georges Pompidou, Prix Marcel Duchamp 2016.

3. Film français réalisé par Pascale Ferran, 2014.

4. L'Encyclopédie de la parole est une compagnie théâtrale. Le spectacle Parlement est sa première création (2009, Fondation Cartier, La Villette, Festival d'Automne, Paris), mise en scène par Joris Lacoste.

\section{INDEX}

Thèmes : Sur le Métier

\section{AUTEURS}

\section{ELSA VIVANT}

Elsa Vivant, elsa.vivant@univ-paris-est.fr , est maître de conférence à l'Université Paris Est Marne la Vallée. Elle a récemment publié :

- Vivant, E., 2021. L'impasse. Scènes de l'urbanisme ordinaire. Créaphis Editions, 213 p.

- Vivant E., 2021. Mettre en scène les territoires et leurs jeux d'acteurs par la création théâtrale. Annales de géographie, $\mathrm{n}^{\circ}$ 739-740, p. 101-121.

- Vivant E., 2021. Changer les pratiques pour sauver des vies. Ressorts et ambiguïtés des programmes d'accompagnement vers le soin mis en œuvre par des polices du Massachusetts face à la crise des opioïdes. Déviance et Société, vol. 45, n 4, p. 613-644. 